Background Efforts to provide screening or treatment of sexually transmitted infections (STIs) among female sex workers (FSWs) require adequate access and uptake of STI-specific health services; this is especially relevant for female sex workers who migrate for work. We examined the patterns of STI clinic use among nonmigrant and migrant FSWs in Karnataka, India.

Methods We used baseline data on 728 non-migrant and 833 migrant FSWs enrolled in a cohort study of FSWs in Karnataka in 2008. The pattern of local (defined as the FSW's place of origin) and destination STI clinic utilisation by migration status of FSWs was examined using univariate and multivariate logistic regression.

Results Overall, $77.9 \%$ and $64.5 \%$ of non-migrant and migrant FSWs had ever attended a local STI clinic $(p<0.001)$. Irrespective of migration status, local sex work was associated with local STI clinic attendance (AOR, 2.9; 95\% CI: 2.3-3.7), and did not vary by other characteristics of sex work. Local clinic attendance was also higher among FSWs who demonstrated awareness of local HIV/STI prevention programmes (AOR 4.7; 95\% CI: 3.3-6.5). Only 33.0\% of migrant FSWs who engaged in local sex work had attended an STI clinic in their destination city, compared to $63.8 \%$ of migrant FSWs who did not participate in local sex work $(p<0.001)$. However multivariate analysis indicated that the association between local sex work and a destination clinic visit was mediated by a lack of awareness of prevention programmes at destination and shorter ( $<1$ month) destination visits. Local clinic attendance remained independently associated with destination clinic visits (AOR 2.8; 95\% CI: 1.8-4.2).

Conclusion Although local STI clinic attendance by FSWs is high, destination clinic visits among migrant FSWs remains low in the presence of local sex work. The findings call for linkages between local and destination HIV/STI prevention programmes to optimise STI-service delivery to migrant FSWs.

\section{P2.151 IMPROVING HAART ENROLLMENT IN ELIGIBLE HIV PATIENTS IN RURAL HAITI}

doi:10.1136/sextrans-2013-051184.0415

A Allen, R Regulus. Zanmi Lasante/PIH, Lascahobas, Haiti

Background In La Colline Health Care Centre, a resource-poor setting in rural Haiti, the HIV/TB department was faced with low performance in HAART enrollment for eligible HIV patients. HIVQUAL (HIV-Quality) reports generated by our EMR (electronic Medical Record) showed 51.2\% ART enrollment for the Oct 2011-March 2012 semester, which further dropped to 33\% by May 2012; meaning that $67 \%$ of patients who needed treatment during that period, did not receive it. A 5-months Quality Improvement project was initiated, from May to September 2012 to find solutions to that problem.

Methods Quality Improvement methods and tools were used to hasten HAART initiation in eligible HIV patients. The project team used the Ishikawa diagram to evaluate the problem and was able to divide the possible causes into 4 groups: those related to people, to environment, to equipment and to procedures. Using Plan-DoStudy-Act cycles, we considered and tested five interventions. Two of them resulted in most of the improvement: Patient tracking by the community team and obtaining $\mathrm{CD} 4$ exam results the same day. Our goal was to reach $80 \%$ HAART enrollment within 6 months.

Results Periodic monitoring of the HAART enrollment indicator via EMR's HIVQUAL report, revelled a progressive improvement in the proportion of eligible HIV patients initiated on HAART; going from $33 \%$ to $43.9 \%, 63.85 \%$ and $84.6 \%$, after respectively the second, third and fourth interventions. The project ended in September 2012, with $90.2 \%$ of eligible HIV patients, receiving HAART.

Conclusion The staff responded positively to the initiative and the changes. The best impact is for the patients who now can benefit from early access to antiretroviral treatment, thus, avoiding some complications of the disease, which can be fatal. This activity contributed to achieving better health care for our HIV patients and a stronger HIV management system.

\section{P2.152 GIVING PATIENTS WHAT THEY WANT: DISCLOSURE ADVICE FOR SEXUALLY TRANSMITTED INFECTIONS AND INFORMATION ON LEGAL REDRESS FOLLOWING INFECTION}

doi:10.1136/sextrans-2013-051184.0416

${ }^{1} E$ Clarke, ${ }^{2}$ O Mansour, 'E Foley, 'R Patel. 'Royal South Hants Hospital, Southampton, UK; '2University of Southampton, Southampton, UK

Background A recent UK prison sentence for transmission of genital herpes simplex virus (HSV) type 2 has caused STI clinics to reassess patient advice on disclosure. Crown Prosecution Service (CPS) guidance, applicable to England and Wales, determines how STI transmission allegations should be handled, including the defensive value of disclosure. Aim: Does advice reflecting CPS guidance alter intended disclosure of STIs and do patients wish to receive this?

Methods Questionnaire study of patients attending a level 3 English STI clinic assessing intended disclosure of STIs and actual disclosure (those with a chronic STI other than HIV) before and after reading CPS guidance on the protective value of disclosure. Likelihood of patients considering legal redress if infected, and whether they wished to receive information on this in STI clinic was reviewed.

Results Of 307 patients the majority intended to disclose an STI diagnosis to regular and casual partners, with generally no significant increase in intended disclosure after disclosure advice. Patients with warts were significantly less likely to disclose to casual than regular partners prior to disclosure advice $(p=0.0138)$, but this difference disappeared after advice. Excluding patients with HSV disclosing to regular partners, intended disclosure was greater than actual disclosure, which was significant in patients with warts $(p=0.0007) .49 \%$ patients would consider legal redress if infected with a chronic STI and $66 \%$ patients would like to recieve information on this in GUM clinic.

Conclusion The study demonstrates that most patients intended to disclose an STI to partners, but that in those with a chronic STI, actual disclosure was lower than this. Advice on the protective value of disclosure did alter disclosure intentions for those with some chronic viral diseases. UK STI clinics should consider raising the issue of the law in relation to the transmission of STIs to reflect patient demand.

\section{P2.153 DELAY OF ENTRY INTO CARE IN HIV POSITIVE INDIVIDUALS}

doi:10.1136/sextrans-2013-051184.0417

${ }^{1} \mathbf{M}$ van Veen, ${ }^{1} \mathrm{~T}$ Heijman, ${ }^{1} \mathrm{P}$ van Leeuwen, ${ }^{2 \mathrm{H}}$ Götz, ${ }^{3} \mathrm{~B}$ van Benthem, ${ }^{4} \mathrm{~S}$ Zaheri, ${ }^{4} \mathrm{~F}$ de Wolf, ' ${ }^{1} \mathrm{H}$ Fennema, ${ }^{3} \mathrm{M}$ van der Sande. 'Public Health Service Amsterdam, Amsterdam, The Netherlands; '2Municipal Health Service Rotterdam-Rijnmond, Rotterdam, The Netherlands; ${ }^{3}$ National Institute for Public Health and the Environment, Bilthoven, The Netherlands; ${ }^{4}$ HIV Monitoring Foundation, Amsterdam, The Netherlands

Background Delay between HIV diagnosis and entry into care may have a negative impact on the individual prognosis and may enable further transmission. Insight into duration and determinants of care-delay is important for public health aimed at reducing ongoing HIV transmission.

Methods Prospective data were collected regionally from consenting patients testing HIV positive at the STI clinics in Amsterdam and Rotterdam, from February 2009-December 2011. Logistic regression analyses were performed to assess risk factors for care delay. Delay of entry into care is defined as a time period of 4 weeks or more between confirmed HIV diagnosis and first consultation at the HIV treatment centre. 
Results In total, 310 participants were included in the study (response $70 \%$ ). The majority were men who have sex with men. Seventy five percent of the study population was referred directly from STI clinic to treatment centre by a clinician or nurse. Thirty percent of participants were delayed: $15 \%$ entered care after 4 weeks and $15 \%$ had not entered care yet. Thus, $70 \%$ of participants entered care within 4 weeks; median time of entry was 9 days (range 0-739 days). In the multivariate model, age below 25 years ( $\mathrm{OR}=3.695 \%$ CI 1.9-6.7) and indirect referral (OR $=3.395 \%$ CI 1.8-6.5) were independent risk factors for care delay, while people with health insurance were less likely to delay ( $\mathrm{OR}=0.395 \%$ CI $0.1-0.9)$. No association was found between care delay and CD4 cell counts or viral load at diagnosis.

Conclusions Younger persons and persons without health insurance are at risk for entering care late after being diagnosed HIV positive. Direct referral from STI clinic to care leads to less delay. Testing of those at risk is not enough to interrupt HIV transmission, entry into care needs to be assured as well. A more active role of the care provider is needed.

\section{P2.154 DETERMINANTS OF UTILIZATION OF A NO-COST HIV TRANSITION CLINIC IN UGANDA: A CROSS SECTIONAL STUDY OF YOUNG ADULTS LIVING WITH HIV/AIDS}

doi:10.1136/sextrans-2013-051184.0418

A Nyabigambo. Makerere University, College of Health Sciences, School of Public Health, Kampala, Uganda

Objective The main purpose of this study was to understand the levels and determinants the HIV Transition Clinic services utilisation by young adults at Infectious Diseases Institute, Kampala, Uganda.

Methods A cross sectional study using quantitative methods at a HTC in a sample of 379 young adults living with HIV/AIDS (YALHA) between the ages of 15-24 years. At analysis utilisation was categorised into two levels; regular (kept all appointment visits) and irregular (missed one or more appointments visits) utilisation. Univariable, bivariable and multivariable logistic regression was used to establish determinants associated with utilisation of the HTC.

Results Of the 379 total respondents, only $32.4 \%$ were regular utilizers of the HTC. There are low levels of regular utilisation of the HTC. Female young adults have better service utilisation rates compared to the males in HTC. The male to female ratio was 1:5. The determinants of HTC regular utilisation were CD4 cell count category of 250-2603/ $\mu \mathrm{l}$ (AOR 0.58, 95\% CI: 0.36-0.95), not currently on ART (AOR 0.27, 95\% CI: 0.15-0.47) and did not receive counselling services (AOR 0.47, 95\% CI: 0.27-0.83).

Conclusion The factors that were associated with reduced the chance of regular use of the HTC were: CD4 cell count between 250-2603/ $\mu \mathrm{l}$; not being on ART; and not receiving HIV counselling services.

Recommendations The young adults in the HIV transition clinic should be screened on a regular basis to detect those with a CD4 cell count of $<350 / \mu \mathrm{l}$ and counselled for early initiation of ART so as to enhance regular HIV transition clinic utilisation. In addition a reminder system like peers, family and mobile phone messages should be set up for young adults so that they are reminded of their appointments hence facilitating regular use of the transition clinic.

\section{P2.155 ADHERENCE MATTERS: A PATIENT-CENTERED ADHERENCE STRATEGY TO ACHIEVE MAXIMUM HEALTH AND QUALITY OF LIFE OUTCOMES FOR PERSONS LIVING WITH HIV/AIDS IN PLAINFIELD, NEW JERSEY}

doi:10.1136/sextrans-2013-051184.0419

L Kudryashova Hernandez. Neighborhood Health Services Corporation, Plainfield, NJ, United States
Background Neighborhood Health Services Corporation (NHSC), an urban community-based not-for-profit ambulatory primary care health centre located in Plainfield, New Jersey, USA, provides HIV care and treatment services to over 350 uninsured, impoverished and minority persons living with HIV/AIDS. To achieve long-term positive health and quality of life outcomes, it is imperative that patients adhere to HIV care and treatment, as well as to prescribed medication regimens.

Methods At NHSC patient-centred adherence efforts begin with a personalised interview. An assessment of patient's clinical and lifestyle indicators is conducted by clinicians followed by formal adherence education video presentations. Other key components of the adherence strategy are detailed discussions of antiretroviral therapies and potential side effects. Patients are provided with culturally and linguistically sensitive printed materials regarding medications and potential side effects. Free Medication boxes are also provided. Weekly monitoring phone calls are conducted by clinical staff to assess compliance and identify adherence challenges. If patients miss appointments, confidential phone calls are placed to reschedule. Treatment Education and Adherence Record is a comprehensive tool that is administered annually to assess adherence, compliance, barriers and any unanticipated outcomes. A multi-disciplinary clinical team led by an Infectious Disease Specialist observes the entire process and monitors patients' boosted compliance with the project and promotion of overall HIV treatment and medications adherence.

Results Resulting from the patient-centred adherence strategy, NHSC observed: $25 \%$ improvement in medical visits no-show rates; $10 \%$ decrease in hospitalisation rates. Furthermore, $62 \%$ of patients maintained CD4 above 200; $52 \%$ of patients have achieved and maintained undetectable viral load.

Conclusions The collected and analysed data suggests that patient-centred adherence efforts help to: reduce no-show rates; minimise side effects and hospitalizations related to missed doses and "drug holidays"; improve and sustain optimal clinical indicators, as well as significantly improve health and quality of life outcomes.

\section{P2.156 PLACE OF NURSES IN THE MANAGEMENT OF PEOPLE LIVING WITH HIV AND AIDS (PLHA)IN DEVELOPING COUNTRIES: CASE OF A REGIONAL HOSPITAL, BURKINA FASO (WEST AFRICA)}

doi:10.1136/sextrans-2013-051184.0420

'Y Sagna, ${ }^{2} \mathrm{M}$ Mano, ${ }^{1,3} \mathrm{~J}$ Y Drabo. ${ }^{1}$ Internal Medicine Department of Yalgado Ouédraogo University Hospital (CHU YO), Ouagadougou, Burkina Faso; ${ }^{2}$ Medicine Department of Tenkodogo Regional Hospital, Tenkodogo, Burkina Faso; ${ }^{3}$ African network of training on HIV (RAF-VIH), Ouagadougou, Burkina Faso

Background Management of people living with HIV and AIDS (PLHA) in the Tenkodogo regional hospital was carried out by a team of 7 nurses and 4 physicians engaged also about their function in the management of non-HIV-infected patients. Only one of these nurses had already received specific training (inter-university graduate) on the management of HIV infection. This team had to manage actually 1213 PLHA including 505 under antiretroviral treatment (ART). Objective We aimed to describe the activities carried out by nurses of this team in the management of PLHA.

Methods We retrospectively reviewed all consultation activities for PLHA by the 7 nurses of the HIV-infected patient's management team at the Tenkodogo regional hospital from January to June 2011. The daily consultation registers of these nurses were our data sources.

Results 2709 consultations of PLHA (any reason confused) were carried out. The consultations mean was 451.5/month. 1886 ART renewals prescription have been made, including 1767 first line and 119 second line treatment. 44 PLHA were newly treated by ART 